\title{
On-line separation of short-lived beryllium isotopes
}

\author{
Ulli Köster ${ }^{a, 1}$, James Barker ${ }^{b, 2}$, Richard Catherall ${ }^{b}$, \\ Valentin N. Fedoseyev ${ }^{c}$, Uwe Georg $^{b}$, Gerhard Huber $^{d}$, Ylva Jading ${ }^{b}$, \\ Ove Jonsson ${ }^{b}$, Mitsuo Koizumi ${ }^{e}$, Karl-Ludwig Kratz ${ }^{f}$, \\ Erich Kugler ${ }^{b}$, Jacques Lettry ${ }^{b}$, Viatcheslav I. Mishin ${ }^{c}$, Helge Ravn $^{b}$, \\ Volker Sebastian $^{d}$, Claire Tamburella ${ }^{b}$, Andreas Wöhr ${ }^{g, 3}$ and the \\ ISOLDE Collaboration ${ }^{b}$
}

${ }^{a}$ Technische Universität München, Physik-Department, 85748 Garching, Bavaria ${ }^{b}$ ISOLDE, CERN, 1211 Genève 23, Switzerland

${ }^{c}$ Institute of Spectroscopy, Russian Academy of Sciences, 142092 Troitsk, Russia

${ }^{d}$ Johannes-Gutenberg Universität, Institut für Physik, 55099 Mainz, Germany

' Japan Atomic Energy Research Institute, 1233 Watanuki, Takasaki, 370-12, Japan

${ }^{f}$ Johannes-Gutenberg Universität, Institut für Kernchemie, 55099 Mainz, Germany

${ }^{g}$ KU Leuven, Instituut voor Kern- en Stralingsfysika, 3001 Leuven, Belgium

\begin{abstract}
With the development of a new laser ionization scheme, it became possible to ionize beryllium efficiently in the hot cavity of the ISOLDE laser ion source. The high target and ion source temperatures enable the release of short-lived beryllium isotopes. Thus all particle-stable beryllium isotopes could be extracted from a standard uraniumcarbide/graphite target. For the first time the short-lived isotopes ${ }^{12} \mathrm{Be}$ and ${ }^{14} \mathrm{Be}$ could be identified at an ISOL facility, ${ }^{14} \mathrm{Be}$ being among the most short-lived isotopes separated so far at ISOLDE. The release time from the UC/graphite target was studied with several beryllium isotopes. Profiting from the element selectivity of laser ionization, the strong and isotopically pure beam of ${ }^{12} \mathrm{Be}$ allowed to determine the half-life to $\mathrm{T}_{1 / 2}=21.34(23) \mathrm{ms}$ and the probability of beta-delayed neutron emission to $P_{n}=0.48_{-0.10}^{+0.12} \%$.
\end{abstract}

\section{Introduction}

Beryllium is a "difficult" element for ISOL (isotope separation on-line) facilities, which is not or only very slowly released. With a vapor pressure of $\approx 10$ mbar at

1) Corresponding author: Tel. +41 22 767-9786, Fax -8990, Email: Ulli.Koster@cern.ch.

2) Present address: University of Greenwich, Woolwich, London SE18 6PF, UK

3) Present address: Oxford University, Department of Physics, Oxford OX1 3PU, UK 
$2000 \mathrm{~K}$ [1], about equal to tin, it is less a problem of evaporation than of adsorption to surfaces. To allow for a fast desorption the whole target-ion source assembly has to be kept at high temperatures. However, the traditional high temperature unit including a surface ion source cannot be applied due to the high ionization potential $(9.32 \mathrm{eV})$ of beryllium. Off-line tests with ${ }^{7} \mathrm{Be}$ in a high temperature plasma ion source MK5 [2] gave an efficiency of $10^{-4}$ at best. About $95 \%$ of the ${ }^{7}$ Be got stuck to cold spots and did not reach the ionizer. It accumulated especially on the surface of the oxide insulators in the plasma source, forming very stable BeO.

For many metals of the groups IIA and IIIA the release speed can be improved considerably by adding $\mathrm{CF}_{4}$ and separating the molecular side band of the monofluoride [3]. However on-line tests with ${ }^{11} \mathrm{BeF}^{+}$and a hot plasma source (all parts above $1800^{\circ} \mathrm{C}$ ) gave only an efficiency of $4 \cdot 10^{-4}$.

A recently presented ECR (electron cyclotron resonance) ion source for the production of ${ }^{7}$ Be beams at TRIUMF [4] reaching an efficiency of 2 to $3 \%$ might be an alternative for off-line produced ${ }^{7} \mathrm{Be}$, but the source body is too cold to allow a rapid release of short-lived beryllium isotopes. Moreover the beam is mainly separated as $\mathrm{BeF}^{+}$and $\mathrm{BeF}_{2}^{+}$. With a heavy target, huge contaminations of isobars like $\mathrm{Ne}^{+}, \mathrm{Na}^{+}, \mathrm{Ar}^{+}, \mathrm{K}^{+}, \mathrm{AlF}^{+}$, etc. are unavoidable and will disturb nuclear spectroscopy experiments strongly due to their comparable lifetimes and decay modes (e.g. $\beta n)$.

\section{Resonance ionization laser ion source}

A new way was opened with resonant photo ionization in a hot cavity laser ion source. For beryllium a new scheme had to be developed including frequency tripling of the dye laser light to reach the first excited state with UV light [5]. An off-line measurement gave an efficiency of 3 to $4 \%$, the on-line yields indicate an even higher efficiency.

\section{Yields}

With a standard ${ }^{4}$ ISOLDE uraniumcarbide/graphite target $(20 \mathrm{~cm}$ long, thickness $52 \mathrm{~g} / \mathrm{cm}^{2}$ of uranium and about $10 \mathrm{~g} / \mathrm{cm}^{2}$ of graphite) an ion implanted ${ }^{7} \mathrm{Be}$ target was produced. It was used for measurements of the astrophysical $\mathrm{S}_{17}$ factor [6]. The same UC/graphite target was used to produce a ${ }^{11}$ Be beam for a collinear laser spectroscopy experiment, where the magnetic moment of ${ }^{11} \mathrm{Be}$ was measured with $\beta$-NMR $[7,8]$. In a target test also the short-lived isotopes ${ }^{12} \mathrm{Be}$ $\left(\mathrm{T}_{1 / 2}=21.3 \mathrm{~ms}\right)$ and ${ }^{14} \mathrm{Be}\left(\mathrm{T}_{1 / 2}=4.4 \mathrm{~ms}\right)$ could be separated for the first time at an ISOL facility. ${ }^{14} \mathrm{Be}$ is among the most short-lived isotopes separated so far at

4) Note that for the production of the long-lived isotopes ${ }^{7} \mathrm{Be}$ and ${ }^{10} \mathrm{Be}$ a "long" (40 cm length) and dense $\left(73 \mathrm{~g} / \mathrm{cm}^{2}\right)$ target from pure graphite was used, giving significantly higher yields when coming into saturation. 
ISOLDE. The given yields (ions per s) are normalized to $1 \mu \mathrm{A}$ of primary proton beam, the average proton current at ISOLDE is between 2 and $4 \mu \mathrm{A}$.

TABLE 1. Measured yields of beryllium isotopes from a standard ISOLDE UC/graphite target.

\begin{tabular}{|r|c|c|c|}
\hline Isotope & Half-life & Yield (ions $/ \mu \mathrm{A} \cdot \mathrm{s}$ ) & Measurement method \\
\hline${ }^{7} \mathrm{Be}$ & $53 \mathrm{~d}$ & $1.4 \cdot 10^{10}$ & Faraday cup \\
${ }^{9} \mathrm{Be}$ & stable & $1.7 \cdot 10^{10}$ & Faraday cup \\
${ }^{10} \mathrm{Be}$ & $1.6 \cdot 10^{6} \mathrm{a}$ & $5.9 \cdot 10^{9}$ & Faraday cup \\
${ }^{11} \mathrm{Be}$ & $13.8 \mathrm{~s}$ & $7 \cdot 10^{6}$ & Faraday cup \& $\beta$ detector \\
${ }^{12} \mathrm{Be}$ & $21.3 \mathrm{~ms}$ & 1500 & $\beta$ detector \\
${ }^{14} \mathrm{Be}$ & $4.4 \mathrm{~ms}$ & 4 & neutron detector \\
\hline
\end{tabular}

\section{Release time}

The release time structure was measured with ${ }^{11}$ Be using the sampling technique (cf. ref. [9]), see fig. 1. This allows to access a time scale of about 0.2 to $30 \mathrm{~s}$.
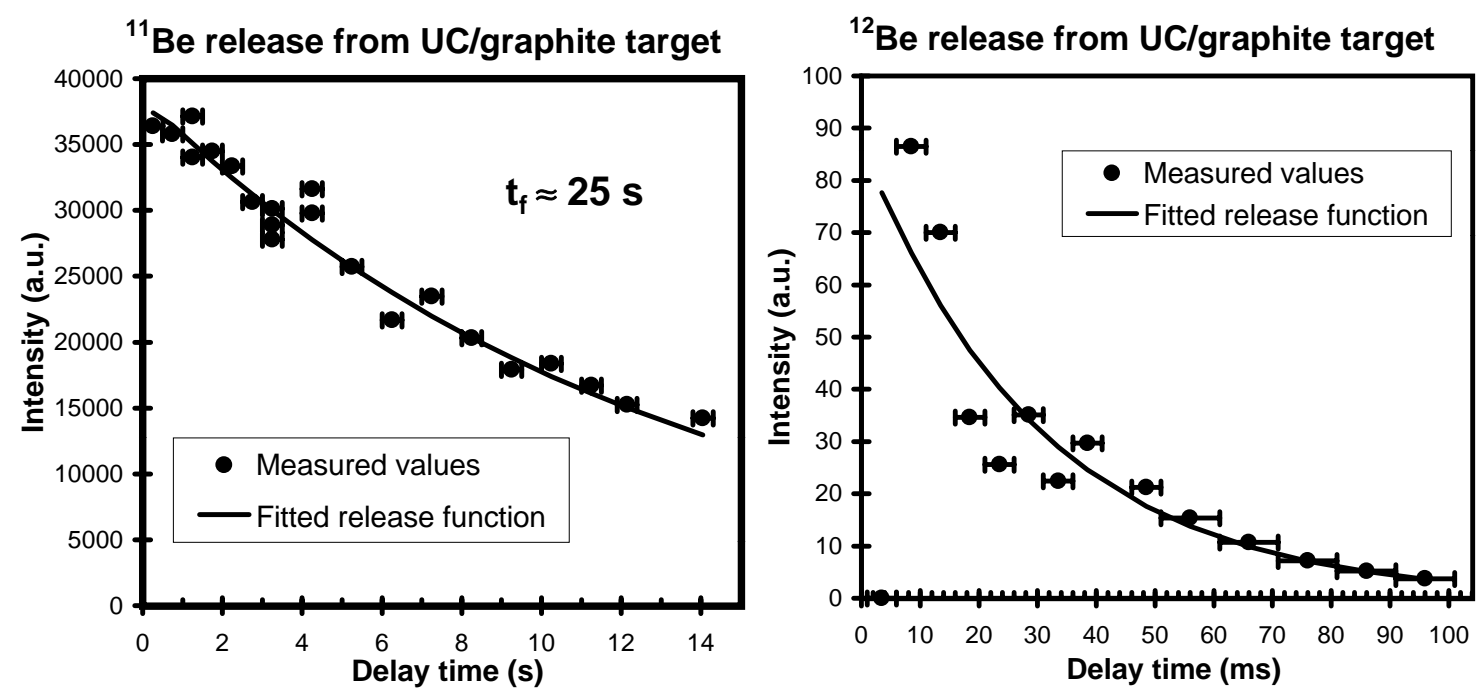

FIGURE 1. Release function of beryllium measured with ${ }^{11} \mathrm{Be}$ and ${ }^{12} \mathrm{Be}$.

For the simplified release function (not taking into account the contribution of a possible slow component):

$$
p(t)=A \cdot\left(1-\exp \left(-t / t_{r}\right)\right) \cdot \exp \left(-t / t_{f}\right)
$$

a fall time $t_{f}$ of about $25 \mathrm{~s}$ can be deduced after correction for the radioactive decay of ${ }^{11} \mathrm{Be}$. The very first part of the release function (up to about $100 \mathrm{~ms}$ ) was monitored with ${ }^{12} \mathrm{Be}$. Despite some fluctuations a rise time $t_{r}$ in the order of $1 \mathrm{~ms}$ can be deduced. This extremely fast rise might indicate a small component 
which is released as a bunch due to the impact of the intense proton pulse. Such a "radiation enhanced release" has already been experienced before, e.g. for noble gases from oxide targets [10]. A slower tail in the minute range can be observed in a Faraday cup from the release of the long-lived radionuclides ${ }^{7} \mathrm{Be}$ and ${ }^{10} \mathrm{Be}$ after switching off the proton beam. A quantitative evaluation is however difficult, since the proton beam contributes to the target heating and to the just mentioned bunched release. During the whole run the target was kept at temperatures above $2000{ }^{\circ} \mathrm{C}$ and the tungsten ionizer cavity above $2300^{\circ} \mathrm{C}$.

\section{Beta-delayed neutron emission}

${ }^{14}$ Be was detected via its beta-delayed neutrons with a $4 \pi$ neutron long counter. ${ }^{3} \mathrm{He}$ proportional counters are arranged in three concentric circles and embedded in a polyethylene matrix for moderation. The neutron detection efficiency is about $30 \%$ in this arrangement. Figure 2 shows the release and decay curve of ${ }^{14} \mathrm{Be}$.

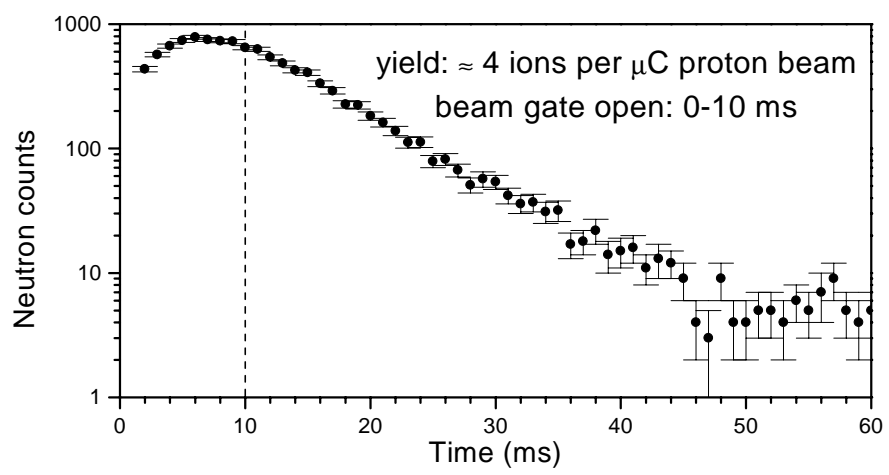

FIGURE 2. Grow-in and decay curve of ${ }^{14} \mathrm{Be}$.

\section{${ }^{12}$ Be decay}

${ }^{12} \mathrm{Be}$ has a $\mathrm{Q}_{\beta}$ value of $11.71 \mathrm{MeV}$. The channel for beta-delayed neutron emission can open at $3.37 \mathrm{MeV}$ excitation energy, thus leaving a rather large window of $8.34 \mathrm{MeV}$ for $\beta n$ decay. Detecting simultaneously the betas with a scintillation detector and the beta-delayed neutrons allows to deduce the probability of betadelayed neutron emission $P_{n}$.

The neutron spectrum can be fitted with a single exponential decay, since any neutron emission in the decay of the daughter ${ }^{12} \mathrm{~B}$ is energetically excluded as well as a $\beta 2 n$-decay of ${ }^{12} \mathrm{Be}$. For the fit of the beta spectrum the half-live of the daughter ${ }^{12} \mathrm{~B}$, which is very precisely known (20.20(2) ms), was kept constant. No boron beam is released directly from the source (the surface ionization of boron is negligible with an ionization potential of $8.3 \mathrm{eV}$ ) but the ${ }^{12} \mathrm{~B}$ produced by ${ }^{12} \mathrm{Be}$ decay in the grow-in phase has to be taken into account. The fits were done with 
the maximum likelihood method to account correctly for the Poisson distribution of the low statistics events [11].

The resulting half-life of $21.34(23) \mathrm{ms}$ is shorter than the older values in literature [12-14], but in agreement with more recent values $[15,16]$.

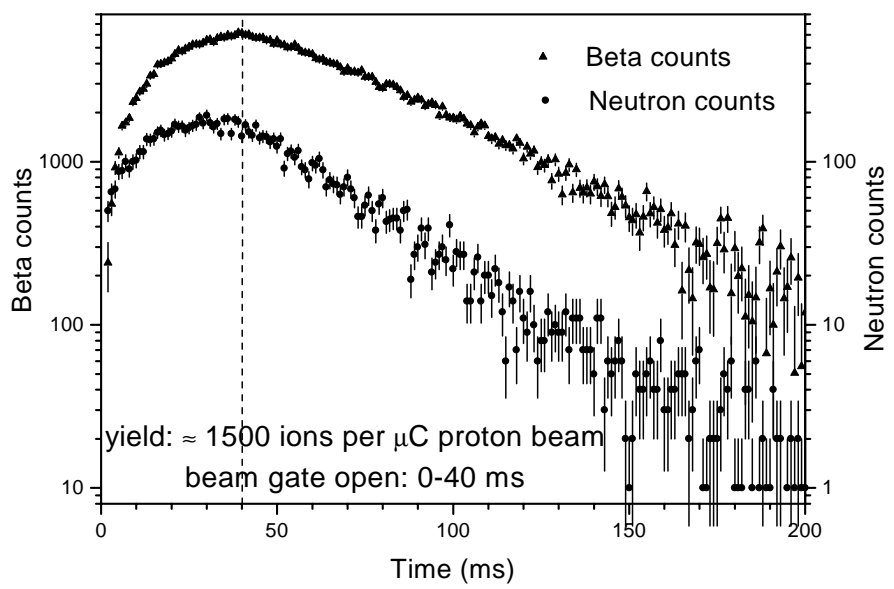

FIGURE 3. Grow-in and decay curve of ${ }^{12} \mathrm{Be}$.

The efficiency ratios of the neutron and beta counters were calibrated on-line with several neutron emitters with known $P_{n}$ values. Since the alignment of these weak ion beams cannot be checked directly with a scanner or Faraday cup, a systematic error arises due to differences in the effective solid angle of the detectors to a possibly shifted implantation position. While this effect is small for the neutron detector it can affect the efficiency for beta detection considerably. An error of about $25 \%$ (relative) for the $P_{n}$ value was derived from the different calibration measurements including the response to various beta and neutron energies of the calibration isotopes. This mainly systematic uncertainty could still be considerably reduced with an enlarged set of calibration data and a careful study of the effective detector efficiencies by Monte Carlo simulations.

The obtained value $\left(P_{n}=0.48_{-0.10}^{+0.12} \%\right)$, which is remarkably low compared to the large energy window available for neutron emission, is in agreement with the upper limits from $[12,13]$ and the value reported in [16]. It disagrees with an older measurement [17] where the beam was probably contaminated with other neutron emitters.

\section{Prospects}

Already now new experiments with beryllium beams are scheduled (detailed nuclear spectroscopy of ${ }^{11} \mathrm{Be},{ }^{12} \mathrm{Be}$ and ${ }^{14} \mathrm{Be}[18]$ ) or planned for the future (measurement of $\mathrm{S}_{17}$ in inverse kinematics at REX-ISOLDE [19]). In our set-up the neutron energy could not be determined accurately. With a different set-up $\left({ }^{3}\right.$ He ionization chambers or a time-of-flight arrangement) it should be possible to determine the populated neutron-emitting levels in ${ }^{12} \mathrm{~B}$. Together with a detailed $\beta \gamma$ spectroscopy 
this should allow to monitor the beta strength distribution over a rather large energy range and to compare it to theoretical predictions, e.g. [21,22]. The laser ion source can even be used to measure directly the isotope shift of the beryllium atoms, see [23].

To perform these experiments a further increase in yield would be very useful. This could be achieved by optimizing the target geometry for fastest release (more compact design). A tantalum foil target which can be heated to very high temperatures (well above $2400{ }^{\circ} \mathrm{C}$ ) could be an alternative to $\mathrm{UC} /$ graphite. By adding $\mathrm{CF}_{4}$ the release time may be improved, but the beryllium-fluoride molecules have to be dissociated in the ionizer tube before laser ionization can be applied.

\section{Acknowledgements}

Thanks for support by the "Beschleunigerlabor der TU und LMU München".

\section{REFERENCES}

1. R.E. Honig and D.A. Kramer, RCA-Review 30 (1969) 285.

2. S. Sundell, H. Ravn and the ISOLDE Collaboration, Nucl. Instr. Meth. B70 (1992) 160.

3. R. Kirchner, Nucl. Instr. Meth. B126 (1997) 135.

4. K. Jayamanna et al., Rev. Sci. Instr. 69 (1998) 756.

5. J. Lettry et al., Rev. Sci. Instr. 69 (1998) 761.

6. Michael Hass et al., contribution to these proceedings.

7. Matthias Keim, contribution to these proceedings.

8. Stefan Kappertz et al., contribution to these proceedings.

9. J. Lettry et al., Nucl. Instr. Meth. B126 (1997) 130.

10. H.L. Ravn et al., Nucl. Instr. Meth. B126 (1997) 176.

11. Y. Jading and K. Riisager, Nucl. Instr. Meth. A372 (1996) 289.

12. D.E. Alburger et al., Phys. Rev. C17 (1978) 1525.

13. J.P. Dufour et al., Z. Phys. A319 (1984) 237.

14. F. Ajzenberg-Selove, Nucl. Phys. A506 (1990) 1.

15. M.S. Curtin et al., Phys. Rev. Lett. 56 (1986) 34.

16. G. Audi, O. Bersillon, J. Blachot and A.H. Wapstra, Nucl. Phys. A624 (1997) 1.

17. A.M. Poskanzer, P.L. Reeder and I. Dostrovsky, Phys. Rev. 138 (1965) B18.

18. Beta-decay study of dripline isotopes of Be, ISOLDE Proposal P99, Spokesperson: G. Nyman

19. Measurement of the ${ }^{7} \mathrm{Be}(\mathrm{p}, \gamma){ }^{8} \mathrm{~B}$ absolute cross section in inverse kinematics, ISOLDE Letter of Intent I20, Spokespersons: C. Rolfs and U. Greife

20. H. Keller et al., Z. Phys. A348 (1994) 61.

21. T. Suzuki and T. Otsuka, Phys. Rev. C56 (1997) 847.

22. T. Suzuki, J. Phys. G24 (1998) 1455.

23. Volker Sebastian et al., contribution to these proceedings. 